\title{
Making pharmaceutical research and regulation work for women
}

\author{
The legacy of male bias within pharmaceutical research, regulation, and commercialisation needs \\ to be rectified, argue Sundari Ravindran and colleagues
}

\section{W}

omen have been excluded from pharmaceutical research for many reasons. ${ }^{1}$ The thalidomide tragedy of the 1960 s prompted a protectionary ban on pregnant women and women of child-bearing age from participating in clinical trials. Other impediments are the perceived complexity and higher costs of studies if women are included, women's unwillingness to participate, and the pervasive treatment of the male body as the norm. ${ }^{1}$ Since the 1980s, there has been strong advocacy in the United States and elsewhere to combat male bias in health research, ${ }^{2}$ and in 1993, the landmark US National Institutes of Health (NIH) Revitalization Act changed the model from excluding women to recommending their inclusion in phase III clinical trials. ${ }^{3}$ The 1995 Beijing Declaration and Platform for Action also condemned medical interventions for women that were based on research carried out predominantly on men.

Major regulatory agencies in the US, Europe, and Canada, whose approvals often influence other countries, have made some positive but fragmented efforts to

\section{KEY MESSAGES}

- Despite increasing evidence of the influence of sex and gender on pharmaceutical outcomes, and some positive country specific regulatory shifts, notable gaps exist in the integration of sex and gender in pharmaceutical research and regulations

- Stronger governance and oversight from regulatory agencies, and commitment by the scientific entrepreneur community, are needed to drive more sex and gender responsive pharmaceutical research, reporting, regulation, and commercialisation

- More women committed to gender equality in leadership and decision making roles in pharmaceutical research and regulation is critical include sex and gender in pharmaceutical regulations (supplementary box 1 on bmj.com). Evidence is increasing of the influence of sex and gender on a wide range of health interventions, including pharmaceutical safety and efficacy profiles ${ }^{4}$ (supplementary box 2). Progressive new policies by health research funding agencies in the US and Canada stipulated that drug development experiments must include an equal balance of male and female cells, tissues, and animals, and that diverse groups of women must be recruited to clinical trials. ${ }^{5} \mathrm{New}$ premarket processes for drug approval by the US Food and Drug Administration, the European Medicines Authority, and Health Canada specifically promoted the inclusion of gender diverse women, older women, and pregnant and lactating women in clinical trials and data reporting (supplementary box 1 ).

Outlooks and practice in pharmaceutical research and regulation are still widely insensitive, however, to the influence of sex and gender on health outcomes. ${ }^{56}$ For instance, the International Council for Harmonisation of Technical Requirements for Pharmaceuticals for Human Use has not recognised the need for specific, standalone guidelines on the inclusion of women, continuing to refer to women as a special subgroup to be considered when appropriate. ${ }^{7}$ Journals inconsistently publish sex disaggregated data on drug efficacy, safety, and toxicity, and industry rarely reports sex or gender differences on product labels. ${ }^{568}$ Fragmented action and resistance perpetuates and extends women's exclusion from timely, safe, and quality of life enhancing therapeutic options across their lives. ${ }^{156}$

\section{Where are the gaps across the pharmaceutical research regulation pathway? \\ Preclinical \\ Gaps in consideration of sex and gender exist at each stage of the research and regu- latory pathway for marketing new pharma- ceuticals (fig 1). Testing of new molecules for product development should include}

experiments on both genetically male and female cells, necessary for early identification of sex differences. ${ }^{6}$ Unsafe dosing recommendations and, consequently, more frequent adverse drug reactions in women, may result from insufficient attention to sex specific pharmacokinetic variations during early preclinical studies. ${ }^{3}$ Animal studies, including those for conditions whose prevalence and morbidity disproportionately affect females, often underrepresent female animals or include male animals only. Thus the opportunity to examine whether therapeutic agents are safe and effective for female populations is undermined. ${ }^{69}$ For instance, different cellular pathways are activated during chronic pain in male and female mice, and findings using male mice do not generalise to female mice. $^{6}$

Although the inclusion of both sexes in preclinical studies has improved in recent years, gaps persist in sex disaggregated reporting and analyses. A survey of 2000 animal studies published in 2010 found that $80 \%$ of studies had male bias. ${ }^{9}$ As of $2016,70 \%$ of biomedical experiments did not report sex as a variable of interest, and less than half of the studies reporting sex included both sexes in the study sample. ${ }^{10}$ After a 2016 NIH requirement for the inclusion of both male and female samples, there have been notable changes in inclusion practice. ${ }^{11}$ In eight of nine disciplines across 720 articles in 34 high impact journals, however, no changes have been seen in sex disaggregated reporting and analysis. ${ }^{11}$

\section{Clinical trials}

Women's representation remains low in clinical trials, particularly in phase I trials $(\approx 22 \%) .{ }^{12}$ Insufficient representation, analysis, and reporting of outcomes in women at different stages of their lives also persist. Pregnant and lactating women continue to be excluded from clinical trials, even at a postmarket stage. ${ }^{13}$ These evidence gaps present difficulties for physicians who need to advise pregnant and breastfeeding women requiring drug treatment. 
Target identification, screening discovery

Finding new or repurposed molecules that can elicit intended biochemical or physiological changes

\section{Premarket}

Preclinical

Laboratory and animal studies to evaluate efficacy and potential risks

Clinical 1-2-3

Trials on humans to identify suitable dose ranges, product effectiveness, and side effects

Market approval

Regulatory submission

Submission of trial data to regulatory authorities to obtain marketing license for specific use

Health technology assessment

Economic, social, and ethical evaluations of product for policy decision making

\section{Postmarket}

Pharmacovigilance

Postmarketing surveillance of adverse events related to use of product

Fig 1 | Pharmaceutical research and regulation pathway

Although recent regulatory changes have led to a general improvement in the inclusion of women in phase III trials, meaningful inclusion of women and analyses of outcomes by sex remain absent or incomplete. ${ }^{8}$ A recent example is emtricitabine/tenofovir alafenamide (Descovy) for HIV pre-exposure prophylaxis. Descovy was approved for the US market in 2019 but only for men with male sex assigned at birth and transgender women. The sponsor company justified the exclusion of people assigned female sex in the DISCOVER trial, citing difficulties in enrolment, resource limitations, and equivocal expectations of reaching meaningful clinical outcomes. ${ }^{14}$

Current guidelines suggest that, at a minimum, women should be represented in trials in proportion to their prevalence in specific health conditions. ${ }^{715}$ This target falls short in serious disease areas, such as cardiovascular conditions ${ }^{15}$ and some cancers. ${ }^{1}$ Of the 40 medicines registered by the FDA in 2019 for conditions affecting both sexes, 16 had $50 \%$ or less representation of women (supplementary box 3).

A focus solely on prevalence will not capture sex differences in the progression of disease or mortality. In the case of erdafitinib, which the FDA approved in 2019 for a type of bladder cancer, women comprised only 18 (21\%) of 87 participants. The justification for this proportion was that men were affected at a threefold to fourfold higher rate despite women having poorer prognostic and survival outcomes even with alternative treatments such as radical cystectomy. ${ }^{1617}$ A different justification is offered in the case of breast cancer in men. Although men comprise less than $1 \%$ of cases of breast cancer, they are more likely to be diagnosed at an older age and advanced stages. ${ }^{18}$ The 2019 draft FDA recommendations for men's inclusion in breast cancer trials specifically note that "low expected accrual rates" are not an acceptable scientific reason for their exclusion, and further data may be necessary "where there is a concern for differential efficacy or safety between men and women." 1418

\section{Regulatory approval and reimbursement}

In recent global efforts to benchmark national pharmaceutical regulation standards, considerations of sex and gender are omitted. ${ }^{19}$ Although guidance exists for industry to submit adequate sex disaggregated data, this requirement is rarely enforced, and few, if any, mechanisms exist for publicly sharing sex disaggregated pharmaceutical data.

Variability in national regulatory decision making on products for women goes unchecked. The US FDA approved the medical abortion pill after four years, in comparison with Japan, where the Ministry of Health took 35 years to approve oral contraceptive pills. In contrast, both the US and Japan registered sildenafil for erectile dysfunction in six months to ensure that the product reached men promptly. ${ }^{20}$

Sex and gender are also rarely considered in value assessments for national drug formularies or insurance benefits packages. ${ }^{21}$ Some analyses show that differences in health outcomes between the sexes may determine the cost effectiveness of interventions. ${ }^{22}$ Additionally, quality of life utility measures, use of medical and health system resources, and work productivity-variables that are included in cost effectiveness calculations-can also differ by sex and gender. ${ }^{22}{ }^{23}$ In the absence of sex disaggregated analyses and reporting of safety, efficacy, and cost data, a sex disaggregated cost effectiveness analysis cannot be performed to inform decision making, potentially resulting in the non-inclusion of products in national or insurance benefits lists that are cost effective for women but not men, and vice versa.

\section{Postmarket use and access}

Evidence gaps for women result in incomplete product information sheets, noninformative labels, and the inability of clinicians to recommend guidelines containing sex specific considerations. ${ }^{24}$ For instance, it is impossible to approve a formal labelled indication of a drug for pregnant women if such women are excluded from clinical trials. This penalises pregnant women by requiring them to pay out of pocket for the unapproved use of an approved drug, depending on the terms of public and private systems of access. ${ }^{1425}$ Such is the case for Descovy, where women's exclusion from clinical trials led to evidence gaps that ultimately limited the approved indication to men only. ${ }^{14}$ Nineteen per cent of new HIV infections in the USA and 75\% in sub-Saharan Africa are in women assigned female sex at birth, but the exclusionary trial design will restrict formal access to this prophylactic option for these women. ${ }^{1426}$

Programme designs of access to medicines that do not take gender into account have negatively affected girls and women. Shortage of the human papillomavirus vaccine for girls in many low and middle income countries, such as Thailand, led to temporary cessation of the national human papillomavirus vaccination programme in $2020 .^{27}$ The reasons for the stoppage included prioritisation of the supply to high income countries, where the vaccine is procured at a higher price and provided to both girls and boys, even though modelling studies suggest only a marginal reduction in prevalence when boys are included. ${ }^{28}$

\section{Postmarket pharmacovigilance}

Women, particularly those aged 18-44, have a higher number of postmarket 
adverse drug reactions and consequently require more related admissions to hospital, whereas men experience more serious and fatal events. ${ }^{29}$ A 2001 US government audit of 10 drugs withdrawn from the market between 1997 and 2000 found that eight out of 10 adverse drug reactions predominantly affected women. ${ }^{30}$ Despite known signals that women and men can have different adverse reactions when using the same pharmaceutical agents, ${ }^{29}$ sex disaggregated data and reporting requirements remain limited, particularly for pregnant women and women in low and middle income countries.

\section{Towards pharmaceutical research and regulation responsive to sex and gender}

Efforts are needed to tackle the lack of regulation, enforcement, and incentives that discourage uptake of practices appropriate to sex and gender. A critical first step is for regulatory agencies to stop framing women as a "subgroup” for analysis. Such framing reinforces justification for excluding women of child-bearing age and pregnant and lactating women from trials and minimises the requirement for adequate representation. ${ }^{56}$ Such deep seated bias spills over into other regulatory processes, delegitimising more aggressive sex and gender focused accountability structures. Initiatives that promote prioritisation of sex and gender responsive research, funding and reporting, institutional norm setting, regulation, and commercial strategy are needed to drive more equitable pharmaceutical outcomes for women (fig 2).

\section{Evidence generation, research funding, and} reporting evaluation criteria for the appropriate proposals, sex differences in discoveries and drug safety information will remain wanting. ${ }^{31} 32$ The Sex and Gender Equity
Until funding agencies consistently adopt integration of sex and gender in research

in Research (SAGER) guidelines provide a roadmap for journal editors to reject manuscripts that do not disaggregate, report, or discuss sex and gender considerations. Without systematic enforcement of these guidelines, however, evidence gaps for women will persist. ${ }^{8}$ Reporting checklists such as CONSORT, PRISM, STROBE, CHEERS, and Cochrane systematic review guidelines require updating, as they include sex only as a subjective subgroup analysis to be optionally considered.

Greater representation of women committed to gender equality in leadership and decision making bodies is well overdue within research teams and at the helm of pharmaceutical companies and regulatory agencies. ${ }^{33} 34$ Women scientists are under-represented in positions of power, are less likely to be laboratory heads and, as a consequence, appear less often as first or last authors in high impact journals. ${ }^{35}$ The absence of women as first authors is especially problematic as studies and initiatives that include women in their leadership team are more likely to consider and report sex differentiated outcomes. ${ }^{1036}$

\section{Regulatory policies and practice}

To promote a shift in research and industry practice, national regulators can make better use of tools already at their disposal, such as enforcement and economic disincentives, which have a track record of success. For example, the inclusion of Japanese participants in global trials increased after Japanese regulators enforced requirements for ethnic representative data in product registration dossiers. ${ }^{37} 38$ Potentially, a similar shift can be driven by increasing the number of women in leadership, who will further the pursuit of gender equality in regulatory science and policies.

However, piecemeal attention by different countries to boost consideration of women in pharmaceutical policies

Evidence generation, research
funding, and reporting
- Greater attention to sex
differences in target
identification, animal studies,
and early human trials
- Stronger journal enforcement
of sex disaggregated
outcome reporting
- More women in leadership
and decision making roles
within research design and
writing teams

Commercialisation and prioritisation of future products

ustry awareness raising prioritised investments in areas of unmet need for

wesearch agenda sett and product priority lists More women committed to gender equality in industry leadership and strategy development will neither achieve global consensus and harmonisation of requirements and approaches, nor repair systematic problems. Improvement might be achieved through international efforts. For example, the inclusion of sex and gender considerations in the World Health Organization's "global benchmarking tool for the evaluation of national regulatory systems" is one possible way of driving the agenda forward, as is the growing focus on equity within health technology assessments. ${ }^{19}$

Drawing on lessons learnt from drugs to treat rare and life threatening conditions and diseases, regulatory agencies should work together to explore and prioritise incentives such as research design support, fee waivers, expedited reviews, brief patent extensions, and tax credits. Regulatory penalties for delayed decision making or rejection based on insufficient sex disaggregated evidence could also be part of an appropriate international strategy. The power to suspend trials that unreasonably exclude women should be enforced, building on existing legislation in some countries such as the US. ${ }^{5}$ Mandatory reporting of sex differences on drug labels is another recommended pressure point. Regulatory agencies should make sex disaggregated data submitted by pharmaceutical agencies freely accessible to the public and the broader academic community.

\section{Sex-gender responsive commercialisation and prioritisation of future products}

Regulation without strategic engagement and collaboration with industry stakeholders is unlikely to be effective. As with Descovy, decisions on women's representation in trials are primarily based on the costs and complexity of recruitment and safety monitoring, with pushback centring on resultant increases in the price and affordability of new medicines. ${ }^{6}$ The choice of regulatory incentives and disincentives should therefore consider the needs and preferences of industry stakeholders. Capacity building and education through early discussion and engagement with innovators, researchers, and industry bodies can help to reduce practices that systematically undervalue or overlook effects on women, leading to commercial decisions not to pursue developments or determine safe and effective dosing. Development of a strategy for the commercialisation of products specifically for women could potentially improve once women who support and advance gender equality are

Fig 2 | Sex-gender responsive research and regulations to support women's access to appropriate medicines and health technology 
better represented in organisational and industry leadership.

A regulatory focus on gender equality has the potential to drive industry trends for prioritised investments in conditions specific to women, research strategies that include analyses and consideration of sex and gender in all therapeutic areas, and alternative pathways for producing commercialised products for women. The high cost of breast cancer therapeutics or biological agents for autoimmune disorders makes them unaffordable in many low and middle income countries. ${ }^{39}$ Industry trends to repurpose older products for which the patent has expired may offer possibilities for providing affordable medicines for conditions that disproportionately affect women. WHO is well placed to facilitate and set this type of research agenda, drawing on past efforts such as the preferred product characteristics and target product profiles in vaccine research. Another example is the FDA's research roadmap, which aims to advance the safety and efficacy and reduce the toxicity of FDA regulated products used by women and expand the FDA's capacity to evaluate products used by women throughout all stages of life and disease. ${ }^{40}$

\section{Product development agenda responsive to sex and gender for all medical interventions}

Adopting a pharmaceutical research and policy agenda (outlined in fig 2 ) responsive to sex and gender is no longer a choice. It is an obligation. Sustainable development goal 5b commits all UN member states to ensuring women's access to all benefits of scientific development and technologies that avert death, disability, and reductions in quality of life, including development of therapeutic agents for the current covid-19 pandemic. Leadership and political will to identify the best way to make pharmaceutical and research policy work for women in a coordinated way will ensure full and equal access to lifesaving treatments and better health outcomes for boys, girls, men, women, and gender diverse people worldwide.

Contributors and sources: The authorship team has expertise and experience on gender and health, health technology assessment, pharmacy, pharmaceutical evaluation and regulation, health systems, public health within high and low and middle income countries, from their work in academic institutions, national regulatory agencies, and the UN system.

Competing interests: We have read and understood BMJ policy on declaration of interests and have no relevant interests to declare. The authors alone are responsible for the views expressed in this article, which does not necessarily represent the views, decisions, or policies of the institutions with which the authors are affiliated.

Provenance and peer review: Commissioned; externally peer reviewed.

This article is part of a series commissioned by The BMJ based on an idea from the United Nations University-International Institute for Global Health and the World Health Organization to mark the 25th anniversary of the adoption of the 1995 Beijing Platform for Action. The BM/ retained full editorial control over external peer review, editing, and publication. United Nations University-International Institute for Global Health paid the open access fees. TK Sundari Ravindran, principal visiting fellow ${ }^{1}$ Yot Teerawattananon, visiting professor ${ }^{2,3}$ Cara Tannenbaum, scientific director ${ }^{4}$ Lavanya Vijayasingham, postdoctoral fellow ${ }^{1}$ ${ }^{1}$ United Nations University-International Institute for Global Health, Malaysia

${ }^{2}$ Health Intervention and Technology Assessment Program (HITAP), Ministry of Public Health, Thailand ${ }^{3}$ Saw Swee Hock School of Public Health, National University of Singapore, Singapore

${ }^{4}$ Institute of Gender and Health, Canadian Institutes of Health Research, Canada

Correspondence to: TKS Ravindran ravindran@unu.edu

\section{(c) (1) \& 8 OPEN ACCESS}

This is an Open Access article distributed under the terms of the Creative Commons Attribution IGO License (https://creativecommons.org/licenses/ by-nc/3.0/igo/), which permits use, distribution, and reproduction for non-commercial purposes in any medium, provided the original work is properly cited.

\section{Check for updates}

1 Yakerson A. Women in clinical trials: a review of policy development and health equity in the Canadian context. Int J Equity Health 2019;18:56. doi:10.1186/s12939-019-0954-x.

2 Parekh A. Women in clinical drug trials: United States Food and Drug Administration update on policies and practices. In: Schenck-Gustafsson K, DeCola PR, Pfaff DW, Pisetsky DS, eds. Handbook of Clinical Gender Medicine. Karger, 2012:466-72. doi:10.1159/000336443.

3 Zucker I, Prendergast BJ. Sex differences in pharmacokinetics predict adverse drug reactions in women. Biol Sex Differ 2020;11:32. doi:10.1186/ s13293-020-00308-5.

4 Mauvais-Jarvis F, Bairey Merz N, Barnes PJ, et al. Sex and gender: modifiers of health, disease, and medicine. Lancet 2020;396:565-82. doi:10.1016/ S0140-6736(20)31561-0.

5 Mazure CM, Jones DP. Twenty years and still counting including women as participants and studying sex and gender in biomedical research. BMC Womens Health 2015;15:94. doi:10.1186/s12905-0150251-9.

6 Tannenbaum C, Day D, Matera Alliance. Age and sex in drug development and testing for adults. Pharmacol Res 2017;121:83-93. doi:10.1016/j. phrs.2017.04.027.

7 International Conference on Harmonisation of Technical Requirements for Registration of Pharmaceuticals for Human Use. Sex-related considerations in the conduct of clinical trials. 2009. https://admin.ich.org/sites/default/files/2019-04/ ICH_Women_Revised_2009.pdf
8 Avery E, Clark J. Sex-related reporting in randomised controlled trials in medical journals. Lancet 2016;388:2839-40. doi:10.1016/S01406736(16)32393-5

9 Zucker I, Beery AK. Males still dominate animal studies. Nature 2010;465:690. doi:10.1038/465690a.

10 Sugimoto CR, Ahn Y-Y, Smith E, Macaluso B, Larivière $V$. Factors affecting sex-related reporting in medical research: a cross-disciplinary bibliometric analysis. Lancet 2019;393:550-9. doi:10.1016/S01406736(18)32995-7.

11 Woitowich NC, Beery A, Woodruff T. A 10-year followup study of sex inclusion in the biological sciences. Elife 2020;9:e56344. doi:10.7554/eLife.56344.

12 Labots G, Jones A, de Visser SJ, Rissmann R, Burggraaf J. Gender differences in clinical registration trials: is there a real problem? $\mathrm{Br}$ J Clin Pharmacol 2018;84:700-7. doi:10.1111/ bcp.13497.

13 Shields KE, Lyerly AD. Exclusion of pregnant women from industry-sponsored clinical trials. Obstet Gynecol 2013;122:1077-81. doi:10.1097/ AOG.0b013e3182a9ca67.

14 Goldstein RH, Walensky RP. Where were the women? Gender parity in clinical trials. N Engl J Med 2019;381:2491-3. doi:10.1056/ NEJMp1913547.

15 Scott PE, Unger EF, Jenkins MR, et al. Participation of women in clinical trials supporting FDA approval of cardiovascular drugs. J Am Coll Cardiol 2018;71. 1960-9. doi:10.1016/j.jacc.2018.02.070.

16 Messer JC, Shariat SF, Dinney CP, et al. Female gender is associated with a worse survival after radical cystectomy for urothelial carcinoma of the bladder: a competing risk analysis. Urology 2014;83:863-7. doi:10.1016/j.urology.2013.10.060.

17 Richters A, Aben KKH, Kiemeney LALM. The global burden of urinary bladder cancer: an update. World J Urol 2020;38:1895-904. doi:10.1007/s00345019-02984-4

18 US Food and Drug Administration. Male breast cancer: developing drugs for treatment. 2020. https://www.fda.gov/regulatory-information/searchfda-guidance-documents/male-breast-cancerdeveloping-drugs-treatment

19 World Health Organization. WHO Global Benchmarking Tool (GBT) for evaluation of national regulatory systems. WHO. 2019. http://www.who.int/ medicines/regulation/benchmarking_tool/en/

20 Hollander I. Viagra's rise above women's health issues: an analysis of the social and political influences on drug approvals in the United States and Japan. Soc Sci Med 2006;62:683-93. doi:10.1016/j.socscimed.2005.03.046.

21 Panteli D, Eckhardt H, Nolting A, Busse R, Kulig M. From market access to patient access: overview of evidence-based approaches for the reimbursement and pricing of pharmaceuticals in 36 European countries. Health Res Policy Syst 2015;13:39. doi:10.1186/s12961-015-0028-5.

22 Schoenbaum M, Sherbourne C, Wells K. Gender patterns in cost effectiveness of quality improvement for depression: results of a randomized, controlled trial. J Affect Disord 2005;87:319-25. doi:10.1016/j. jad.2005.03.018.

23 Frick KD, Jones AS. Gender bias in economic evaluation methods: quality of life and family role effects. Womens Health Issues 2008;18:4-6. doi:10.1016/j.whi.2007.11.003

24 Tannenbaum C, Norris CM, McMurtry MS. Sexspecific considerations in guidelines generation and application. Can J Cardiol 2019;35:598-605. doi:10.1016/j.cjca.2018.11.011.

25 Hamel S, McNair DS, Birkett NJ, Mattison DR, Krantis A, Krewski D. Off-label use of cancer therapies in women diagnosed with breast cancer in the United States. Springerplus 2015;4:209. doi:10.1186/ s40064-015-0981-z. 
26 UNAIDS. Global HIV \& AIDS statistics-2020 fact sheet. 2020. http://www.unaids.org/en/resources/ fact-sheet

27 Teerawattananon Y. Who cares for global health? The need to take vaccine policy beyond borders. Proceedings of the Vaccinology for Clinical and Public Health Practice Workshop. Faridabad, India. Nonthaburi: Health Intervention and Technology Assessment Program. 2019.

28 Datta S, Pink J, Medley GF, et al. Assessing the cost-effectiveness of HPV vaccination strategies for adolescent girls and boys in the UK. BMC Infect Dis 2019;19:552. doi:10.1186/s12879-019-4108-y.

29 Watson S, Caster O, Rochon PA, den Ruijter H. Reported adverse drug reactions in women and men: aggregated evidence from globally collected individual case reports during half a century. EClinicalMedicine 2019;17:100188. doi:10.1016/j. eclinm.2019.10.001.

30 US Government Accountability Office. Drug safety: most drugs withdrawn in recent years had greater health risks for women. 2001. https://www.gao.gov/ assets/100/90642.pdf
31 European Commission Research and Innovation. Toolkit gender in EU-funded research. 2011. https://www.ki.si/fileadmin/user_upload/ KINA24840ENC 002.pdf

32 Canadian Institutes of Health Research. Sex, gender and health research. 2019. https://cihr-irsc. gc.ca/e/50833.html

33 Schiebinger L. Women's health and clinical trials. J Clin Invest 2003;112:973-7. doi:10.1172/ $\mathrm{JCl} 200319993$.

34 Davies SE, Harman S, Manjoo R, Tanyag M, Wenham C. Why it must be a feminist global health agenda. Lancet 2019;393:601-3. doi:10.1016/S0140 6736(18)32472-3.

35 Coiro P, Pollak DD. Sex and gender bias in the experimental neurosciences: the case of the maternal immune activation model. Transl Psychiatry 2019;9:90. doi:10.1038/s41398-019-0423-8.

36 Nielsen MW, Andersen JP, Schiebinger L, Schneider IW. One and a half million medical papers reveal a link between author gender and attention to gender and sex analysis. Nat Hum Behav 2017;1:791-6. doi:10.1038/s41562-017-0235-x.
37 Asano K, Uyama Y, Tohkin M. Factors affecting drug-development strategies in Asian global clinical trials for drug approval in Japan. Clin Transl Sci 2018:11:182-8 doi:10.1111/cts.12520.

38 Bancroft C. Meeting clinical trial data requirements in Asian markets. 2020. https://www.clinicalleader. $\mathrm{com} / \mathrm{doc} / \mathrm{meeting}$-clinical-trial-data-requirements-inasian-markets-0001

39 Rannan-Eliya RP, Anuranga C, Manual A, et al. Improving health care coverage, equity, and financial protection through a hybrid system: Malaysia's experience. Health Aff (Millwood) 2016;35:838-46. doi:10.1377/hlthaff.2015.0863.

40 US Food and Drug Administration. Women's health research roadmap. FDA. 2020. https://www.fda.gov/ media/97501/download

\section{Supplementary boxes showing regulatory changes and sex differences in drug trials}

Cite this as: $B M J$ 2020;371:m3808

http://dx.doi.org/10.1136/bmj.m3808 